\title{
A DOUBLE BLIND RANDOMIZED CONTROLLED PRELIMINARY TRIAL TO OPTIMIZE NEEDLE GAUGE FOR VACCINATION IN INFANTS
}

\author{
H.K. Pemde, N. Nirupam, A.K. Dutta \\ Pediatrics, Lady Hardinge Medical College, New Delhi, India
}

Background and aims: Several guidelines recommend various needle gauges for vaccinating infants. Good quality evidence is hardly available for these recommendations. This pilot study was done to find optimum needle gauge for minimizing adverse reactions following DPT and Hepatitis-B vaccines.

Methods: Infants of $6-10$ weeks of age were randomized to receive DPT on right thigh and Hepatitis-B on left thigh with 22, 23 or 24 gauge needles. Outcome was observed in terms of injection site reactions (redness, swelling and tenderness) and systemic reactions (fever, vomiting, lethargy, persistent crying, and irritability) on subsequent four days. A convenience sample of 50 infants was taken in each group.

Results: Out of 373 infants screened, 50 each were included in 3 groups. Three groups had similar demographic variables (sex, birth weight, age, weight or length).

Intergroup comparison for injection site and systemic reactions did not reveal any significant differences. There was no significant change in family routine in all the 3 groups but $8-12 \%$ of parents complained of minor changes like apprehension, anxiety and monetary losses and $<2 \%$ of major changes in family routine.

Relative risks of having local or systemic reactions following intramuscular injections using 22, 23 or 24 gauge needles were slightly less with gauge 22 but the $95 \%$ confidence intervals crossed 1 and hence were insignificant.

Conclusion: The local and systemic reactions are not different when different gauge needles of same length are used to administer the vaccines (DPT and Hepatitis-B) intramuscularly in children at 6-10 weeks of age. 